\title{
Calcium deficiency-induced secondary hyperparathyroidism and osteopenia are rapidly reversible with calcium supplementation in growing rabbit pups
}

\author{
Meenal Mehrotra ${ }^{1}$, Sushil K. Gupta ${ }^{1}$, Krishan Kumar ${ }^{1}$, Pradeep K. Awasthi ${ }^{1}$, Manoj Dubey ${ }^{1}$, Chandra M. Pandey ${ }^{2}$ \\ and Madan M. Godbole ${ }^{1 *}$ \\ ${ }^{1}$ Department of Medical Endocrinology, Centre for Endocrine Sciences, Sanjay Gandhi Post Graduate Institute of Medical Sciences, \\ Raebareli Road, Lucknow 226014, India \\ ${ }^{2}$ Department of Biostatistics, Sanjay Gandhi Post Graduate Institute of Medical Sciences, Raebareli Road, Lucknow 226014, India
}

(Received 4 April 2005 - Revised 18 October 2005 - Accepted 20 October 2005)

\begin{abstract}
The reversibility of osteopenia secondary to isolated Ca deficiency (CaDef) is still not clear. We studied the effect of severe CaDef on Ca homeostasis and bone accrual in a 'hypercalcaemic' animal, the rabbit, during the post-weaning period and its reversibility on Ca supplementation. Male Belgian 5-week-old rabbit pups were fed CaDef diet $(0.026 \% \mathrm{Ca})$ for 10 weeks. As compared with those fed with a normal chow diet $(0 \cdot 45 \% \mathrm{Ca})$, CaDef pups developed significant hypocalcaemia $(P<0 \cdot 05$ ), hypocalciuria (urinary Ca 76 (SEM 12) v. 17 (SEM 1) mg/l; $P<0.005$ ), hypophosphataemia (serum inorganic P 100 (SEM 6) v. 65 (SEM 4) $\mathrm{mg} / \mathrm{l} ; P<0.005$ ), secondary hyperparathyroidism (SHPT) (serum intact parathyroid hormone human equivalent 18.2 (SEM 1.4) $v$. 125.0 (SEM 4.5) pg/ml; $P<0.001$ ) and elevated serum calcitriol levels (34.0 (SEM 3.9) v. 91.0 (SEM 1.0) pg/ml; $P<0.005$ ). Elevated urinary C-terminal telopeptide of class I collagen $(P<0.005)$ and total serum alkaline phosphatase $(P<0.005)$ suggested increased bone turnover. There was a significantly lower gain in bone mineral density (BMD) and bone mineral content (BMC) in the whole body and lumbar spine in vivo, and various sub-regions of the femur and tibia in vitro. Supplementation of adequate $\mathrm{Ca}(0.45 \% \mathrm{Ca})$ after 15 weeks on the normal diet resulted in rapid catch-up growth, and resolution of SHPT. Rapid gain in various BMD and BMC parameters continued at 30 weeks of age, and both were comparable with those in rabbits on a normal diet. We conclude that Ca deficiency-induced SHPT and poor bone accrual in growing rabbit pups are rapidly reversible with Ca supplementation. The present study indicates that early intervention may be a more appropriate window period for human nutritional corrective measures.
\end{abstract}

Hypercalcaemic animal models: Bone mineral density: Bone mineral content: Rickets

The hypothesis that low Ca intake could be a limiting factor for achievement of peak bone mass has derived support from various epidemiological and experimental studies (for references, see Pettifor \& Moodley, 1997). Studies from Africa and India have documented rickets and osteomalacia in children on extremely low dietary $\mathrm{Ca}$ intakes, despite having adequate vitamin $\mathrm{D}$ status (Pettifor et al. 1978, 1981a,b; Marie et al. 1982; Eyberg et al. 1986; Okonofua et al. 1991; Bhimma et al. 1995; Oginni et al. 1996; Fischer et al. 1999; Balasubramanian et al. 2003; Rajeshwari et al. 2003). High prevalence of hypocalcaemia and elevated alkaline phosphatase (ALP) concentrations in black school children without evidence of rickets or bone deformities has also been reported (Pettifor et al. 1979).

Although there are a few studies documenting the effect of a low-Ca diet in animal models during the active growth period (Gilsanz et al. 1991; Norris et al. 2001; Iwamoto et al. 2004; Bas et al. 2005), there has been only one intervention study published so far (Peterson et al. 1995). In this study female rats were kept on a low $\mathrm{Ca}$ intake $(0.25 \%$; mild deficiency) for 20 weeks starting from the time of weaning at the 4th week. The reversibility of the effect of $\mathrm{Ca}$ deficiency on peak bone mass was studied after the introduction of either 0.5 or $1.0 \%$ $\mathrm{Ca}$ intake at the 24th week until 37 weeks. The results showed that low $\mathrm{Ca}$ intakes through adolescence have non-reversible deleterious effects on peak bone mass, whereas higher intakes promote greater peak bone mass and provide the potential protection from age-related bone loss. The important question not addressed before, however, is the extent to which catch-up skeletal mineralisation can take place on early intervention following a post-infancy period of severe Ca deficiency. Since deleterious effects of both short-term and long-term low $\mathrm{Ca}$ intake of 0.1 and $0.15 \%$ (moderate deficiency) respectively have been shown in both growing female rats and male rabbit models (Gilsanz et al. 1991; Iwamoto et al. 2004), the important question of the extent to which severe Ca deficiency in infancy can be reversed with earlier intervention is not known. The aim of the present study, therefore, is to investigate the effect of severely restricted dietary $\mathrm{Ca}$ intake $(0.026 \%)$ on growth and bone health in post-weanling male rabbit pups and to elucidate the mechanisms governing the attainment of peak bone mass by the analysis of calcitropic hormones and biochemical markers. Furthermore, the present study tested the hypothesis whether 
early intervention results in better bone accretion, peak bone mass attainment and temporal pattern achievement relevant to early childhood Ca-deficiency rickets eradication initiatives. The choice of the young, still-growing rabbit as an appropriate animal model for peak bone mass studies has recently been well established due to similarities with human patterns of bone accretion and dual-energy X-ray absorptiometry (DXA)-derived normative data for bone mineral content (BMC) and bone mineral density (BMD) (Norris et al. 2001). The present study reports that short-term severe $\mathrm{Ca}$ deficiency in developing rabbits induces hypocalcaemia, secondary hyperparathyroidism, increased bone turnover in favour of bone resorption and significantly lesser accrual of BMD in the whole animal and in long bones. Most importantly, these changes are reversible with early $\mathrm{Ca}$ supplementation in contrast to the earlier reported irreversibility in growing rats (Peterson et al. 1995) and hence define early intervention as a more appropriate window period for human nutritional corrective measures.

\section{Materials and methods}

Seventy-two Belgian male weanling rabbit pups, aged 5 weeks, were procured from the Central Drug Research Institute, Lucknow, India. The rabbit pups were separately housed in metabolism cages at $24^{\circ} \mathrm{C}$ on a $12 \mathrm{~h}$ light and dark cycle. The Institute's Ethical Committee for Animal Experiments approved the experimental protocol. The rabbits were randomised into two groups. In group I (control group; $n$ 36), the rabbit pups were fed a normal chow diet (Ca content $0.45 \%)$ and tap water (Ca content $35.4 \mathrm{mg} / \mathrm{l}$ ) ad libitum until 30 weeks of age. The recommended dietary Ca intake in rabbits is $0.45 \%$ (Gilsanz et al. 1991). In group II (Ca-deficient group, $n$ 36), the rabbit pups were fed a Ca-deficient diet (Ca content $0.026 \%$ ) and deionised water ad libitum until 15 weeks of age. For group III (Ca-replenished group, $n 12$; recovery experiment), after 15 weeks, group II rabbits were fed the same chow diet and tap water ad libitum as group I until 30 weeks of age (30 weeks is the age for skeletal maturity in rabbits) (Gilsanz et al. 1988, 1991).

Growth, behaviour and mobility were observed every 2 weeks until 15 weeks, then every 5 weeks until 30 weeks. At each time interval, blood was collected by venepuncture from the marginal ear vein, serum was separated and total $\mathrm{Ca}$, inorganic $\mathrm{P}(\mathrm{iP})$, total ALP, albumin and creatinine were estimated on the same day. Serum samples were stored at $-70^{\circ} \mathrm{C}$ until the time of other assays. Urine was collected and refrigerated in plastic bottles with toluene as preservative. Faecal matter was collected, weighed and refrigerated. A skiagram of each whole rabbit was obtained. Four rabbits were killed at each time interval, and femur and tibia were dissected out for in vitro BMD and $\mathrm{Ca}$ content.

\section{Biochemical and hormonal estimations}

Serum total Ca, spot urinary $\mathrm{Ca}$ (UCa), serum albumin, serum iP, urinary creatinine (colorimetric method) and total ALP (colorimetric kinetic method) were estimated by commercially available kits (Sigma Diagnostics, St Louis, MO, USA). Serum total Ca was corrected for albumin to calculate corrected serum $\mathrm{Ca}$ (CCa). Faecal and bone $\mathrm{Ca}$ were measured by the colorimetric method after ashing and extracting $\mathrm{Ca}$ with $1 \mathrm{M}-\mathrm{HCl}$. Serum calcidiol (RIA; DiaSorin, Stillwater, MN, USA), calcitriol (RIA;
DiaSorin), intact parathyroid hormone (iPTH; human equivalent) (IRMA; Diagnostic Systems Laboratory, Webster, TX, USA), and urinary C-terminal telopeptide of type I collagen (Crosslaps ${ }^{\mathrm{TM}}$; ELISA; Diagnostic Systems Laboratory) were measured. The sensitivity of serum calcidiol, calcitriol, iPTH and urinary Crosslaps ${ }^{\mathrm{TM}}$ were $1.5 \mathrm{ng} / \mathrm{ml}, 4 \mathrm{pg} / \mathrm{ml}, 6 \mathrm{pg} / \mathrm{ml}$ and $50 \mu \mathrm{g} / \mathrm{l}$ respectively. The interassay and intra-assay $\mathrm{CV}$ for calcidiol were 12 and $10.5 \%$, for calcitriol 12.5 and $11 \%$, for iPTH 6.5 and $5 \%$, and for Crosslaps ${ }^{\mathrm{TM}} 4.7$ and $3.5 \%$.

\section{Areal bone mineral density and bone mineral content quantification}

Areal BMD (aBMD) and BMC were measured by DXA (Hologic QDR 4500 A; Hologic Inc., Waltham, MA, USA) using small animal software. The instrument was calibrated with a small animal step phantom daily. The short-term drifts of DXA measurements were assessed by measuring two rabbits and two femora five times on the same day. The $\% \mathrm{CV}$ for in vivo and in vitro measurement were 0.56 and $0.61 \%$ respectively. The aBMD and BMC results are expressed as $\mathrm{g} / \mathrm{cm}^{2}$ and $\mathrm{g}$ respectively.

In vivo measurements. Anaesthetised (diethyl ether) rabbits were scanned in a supine position with the appendages secured flat to the table by a thin tape. The aBMD and BMC measurements were analysed in the whole body (WB) and the lumbar spine (LS) region $\left(\mathrm{L}_{1}-\mathrm{L}_{7}\right)$.

In vitro measurements. Femora and tibia were dissected out and adherent soft and fatty tissue were mechanically removed. Individual bones were placed in water and scanned. All the bones were measured on the same day. Sub-regional analyses of femora were performed at the femoral neck, and the proximal, mid and distal third regions of the femur. Similarly, the subregional analyses of tibia were done at the proximal, mid and distal third regions.

\section{Body composition analysis}

Body composition analysis was performed by DXA. The percentage contents of lean mass, fat and BMC were calculated.

\section{Statistical analysis}

The data were analysed using SPSS for Windows 9.0 software (SPSS Inc., Chicago, IL, USA). The normality of data was analysed by the Kolmogorov-Smirnov test with Lilliefor's significance correction. Except UCa and Crosslaps ${ }^{\mathrm{TM}}$ levels, all other parameters were normally distributed. The data are expressed as mean values with their standard errors. The comparisons of various parameters were performed between group I and group II from 5 weeks to 15 weeks and between group I and group III from 15 to 30 weeks. Normally distributed parameters were compared by Student's $t$ test while data not normally distributed were compared using the Mann-Whitney test. $P$ values less than 0.05 were considered significant.

\section{Results}

Baseline

There was no significant difference in mean weight, serum $\mathrm{Ca}$, albumin, $\mathrm{CCa}$, iP, ALP, calcidiol, calcitriol, and iPTH (human 
equivalent), UCa, Crosslaps ${ }^{\mathrm{TM}}$ and creatinine excretion, faecal Ca excretion, and bone $\mathrm{Ca}$ contents $(P>0.05)$ between groups I and II. Mean $\mathrm{aBMD}$ and $\mathrm{BMC}$ of the $\mathrm{WB}$ in vivo, and $\mathrm{BMD}$ and $\mathrm{BMC}$ of various regions of tibia and femur in vitro were similar in both the groups $(P>0 \cdot 05)$.

\section{Group I v. group II (5-15 weeks)}

The rabbits in group I showed a progressive increase in weight, were active with normal gait, posture and had normal, shiny fur coats (Fig. 1). In contrast, group II rabbits had a significantly smaller increase in weight (Fig. 2(A)) and loss of body hair (Fig. 1). The rabbits had reduced mobility and difficulty in walking. Two rabbits died at 13 weeks due to severe hypocalcaemia and three rabbits sustained spontaneous hindlimb fractures.

In group I, serum $\mathrm{CCa}$, albumin, $\mathrm{iPTH}$, calcidiol and calcitriol levels were almost constant from 5 to 30 weeks. There was a significant decline in serum iP from 5 to 7 weeks ( 5 weeks 133 (SEM 14) $v .7$ weeks 88 (SEM 5) mg/l; $P<0 \cdot 05$; Fig. 2(C)) and thereafter a gradual decline until 30 weeks (54.3 (SEM 1.8) mg/l). Serum total ALP reduced progressively from 5 to 30 weeks (Fig. 2(E)). UCa excretion was low at 5 weeks (18 (SEM 2) mg/l), progressively increased until 11 weeks (89 (SEM 17) $\mathrm{mg} / \mathrm{l})$, with a significant increase at 15 weeks onwards (20 weeks 214.2 (SEM 23.4) mg/l; $P<0.05$ ). At 30 weeks, the UCa value was 237.2 (SEM 7.8) $\mathrm{mg} / \mathrm{l}$, indicating that hypercalciuria in rabbits develops at 20 weeks (Fig. 3(A)). There was a progressive increase in faecal $\mathrm{Ca}$ excretion (Fig. 3(C)). Urinary Crosslaps $^{\mathrm{TM}}$ excretion was stable until 15 weeks, then had a significant increase at 15 weeks onwards indicating high bone turnover status in adult rabbits (15 weeks 0.44 (SEM 0.01) v. 30 weeks 1.27 (SEM 0.13) $\mu \mathrm{g} / \mathrm{mm}$-creatinine; $P<0.05$; Fig. 3(B)).

In contrast, rabbits in the Ca-deficient group (group II) had a progressive decline in serum CCa levels from 7 until 11 weeks (5 weeks 125 (SEM 9) v. 11 weeks 118 (SEM 6) mg/l; $P<0.05$ ). However, thereafter, the $\mathrm{Ca}$ levels normalised under the influence of increasing serum iPTH concentrations (Fig. 2(B)). Serum CCa was significantly lower at the 7 th, 9 th and 11 th weeks as compared with those in group I $(P<0 \cdot 05)$. Two rabbits suffered

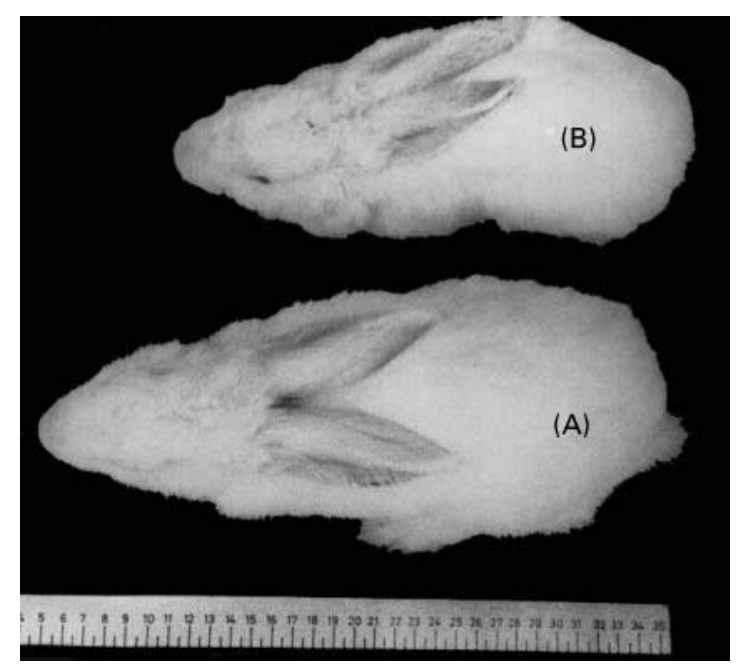

Fig. 1. Appearance of the rabbits in group I (A) and group II (B) at 15 weeks showing retarded physical growth in the Ca-deficient rabbit. For details of animals and procedures, see p. 583. sudden death due to severe hypocalcaemia at 13 weeks. The serum CCa levels $1 \mathrm{~d}$ before their deaths were 55 and $75 \mathrm{mg} / \mathrm{l}$, respectively. Hypophosphataemia was observed at 13 and 15 weeks $(P<0.001$; Fig. $2(\mathrm{C}))$. There was a progressive increase in serum iPTH and total ALP levels at 7 weeks onwards until 15 weeks (iPTH, 5 weeks 18.2 (SEM 1.4) v. 15 weeks 125.0 (SEM 4.5) $\mathrm{pg} / \mathrm{ml}, P<0.001$, Fig. 2(D); ALP, 5 weeks 132.9 (SEM 8.0) v. 15 weeks 192.9 (SEM 5.1) IU/l, $P<0.005$, Fig. 2(E)). Both parameters were significantly higher at $9-15$ weeks in group II rabbits as compared with those in group I (Fig. 3(D) and Fig. 3(E)). Evidence of Ca deficiency was apparent in view of significantly lower urinary and faecal $\mathrm{Ca}$ excretion $(P<0.05$; Fig. 3(A) and 3(C) respectively). With comparable serum calcidiol levels between group I and group II (5 weeks group I $1.9($ SEM 0.2) $v$. group II $2.4($ SEM 0.5$) \mathrm{ng} / \mathrm{ml}, P>0.05$; 15 weeks group I 2.4 (SEM 0.4) $v$. group II $2.8($ SEM 0.2$) \mathrm{ng} / \mathrm{ml}$, $P>0.05$ ), we observed a sharp increase in serum calcitriol levels from 5 to 15 weeks (5 weeks 34.0 (SEM 3.9) v. 15 weeks 91.0 (SEM 1.0) pg/ml; $P<0.005$; Fig. 2(F)). There was a progressive increase in urinary Crosslaps ${ }^{\mathrm{TM}}$ excretion 7 weeks onwards (7 weeks 0.26 (SEM 0.02) v. 15 weeks 1.34 (SEM 0.13 ) $\mu \mathrm{g} / \mathrm{mM}$-creatinine; $P<0.005$; Fig. 3(B)).

In vitro bone analysis. The weight and $\mathrm{Ca}$ contents of isolated femur and tibia increased progressively in group I rabbits. In group II, the weight and $\mathrm{Ca}$ contents of both femur and tibia did not increase until 15 weeks of age, and thus were significantly lower as compared with those in group I $(P<0.005)$ (data not shown).

Skiagrams. As compared with group I, skiagrams of group II rabbits showed marked cortical thinning. Diaphyseal fractures of long bones in three rabbits, complete as well as greenstick, were also observed (Fig. 4).

Bone mineral density. Group I rabbits showed a progressive increase in WBBMD (Fig. 5(A)), WBBMC (data not shown), LSBMD (Fig. 5(B)) and LSBMC (data not shown) in vivo. In vitro sub-regional analysis of isolated femur (proximal, mid and distal third and femoral neck) and tibia (proximal, mid and distal third) showed progressive increases in BMD and $\mathrm{BMC}$ in each region from pup stage to adulthood (Figs. 6 and 7).

In contrast, Ca-deficient rabbits had an initial decline in WBBMD (Fig. 5(A)), while there was no increase in WBBMC until 15 weeks (data not shown). This shows markedly diminished $\mathrm{Ca}$ accrual in the bone in Ca-deficient states. The in vivo LSBMD and LSBMC (data not shown) showed no change in group II rabbits from 5 to 15 weeks (BMD 5 weeks 0.1318 (SEM 0.004) v. 15 weeks 0.1209 (SEM 0.002) g/ $\mathrm{cm}^{2}$ ). Hence, LSBMD was significantly lower at 11-15 weeks when compared with group I rabbits $(P<0.05$; Fig. 5(B)). In vitro densitometric measurements showed no significant accrual of BMD and BMC in whole tibia and whole femur (data not shown). Similar poor accrual of BMD and BMC was seen in sub-regional analysis of both femur and tibia (Figs. 6 and 7). All these densitometric parameters (sub-regional BMD and BMC) were significantly lower as compared with group I at 9 weeks onwards. This indicates a significant impact of $\mathrm{Ca}$ deficiency within 4 weeks of the start of the Ca-deficient diet.

In group II, no change was observed in the percentage fat and percentage lean mass at any time interval between the groups while percentage BMC was significantly lower at 9-13 weeks $(P<0.005)$ in group II as compared with group I (data not shown). 


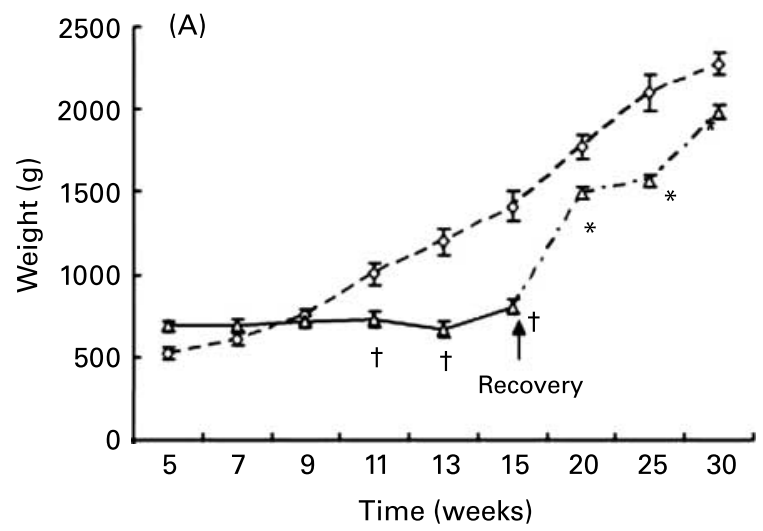

(B)
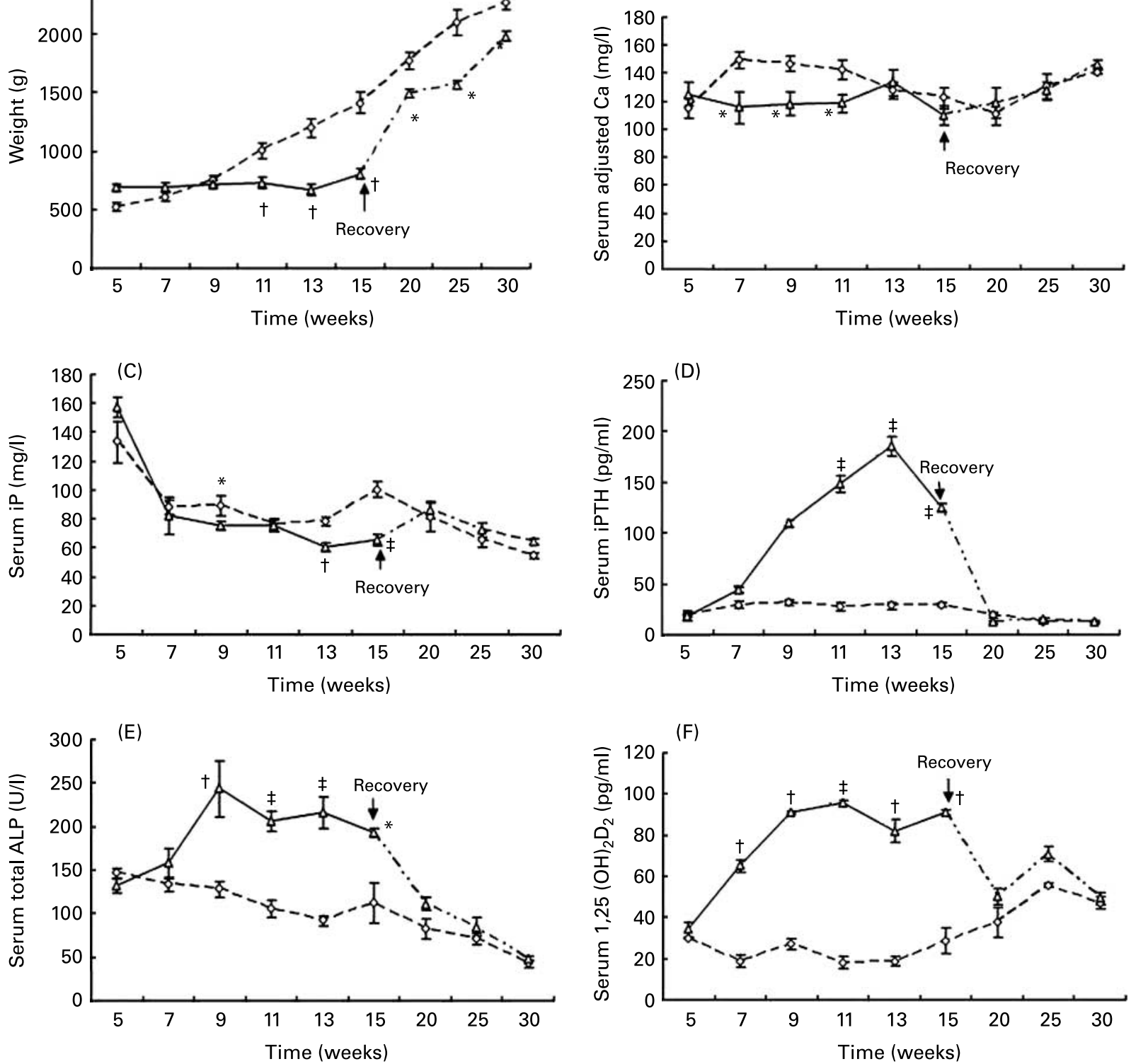

$(\mathrm{F})$

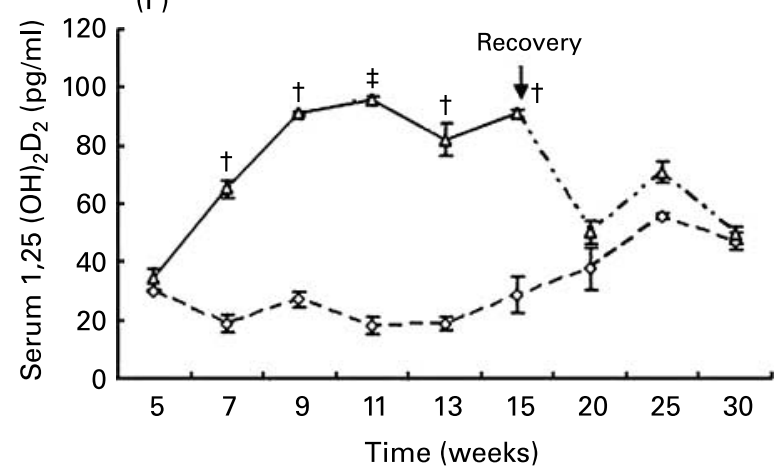

Fig. 2. Serial changes in (A) body weight, $(B)$ serum adjusted $\mathrm{Ca},(\mathrm{C})$ serum inorganic $P(\mathrm{iP})$, (D) serum intact parathyroid hormone (iPTH; human equivalent), (E) serum alkaline phosphatase (ALP) and $(F)$ serum 1,25 dihydroxycholecalciferol $\left(1,25(\mathrm{OH})_{2} \mathrm{D}_{3}\right)$ in group $1(-\diamond-)$, group II $(-\Delta-)$ and group III $(--\downarrow-)$ at different time intervals. Data are expressed as mean values, with their standard errors represented by vertical bars. Mean values were significantly different: ${ }^{\star} P<0.05$, $\dagger P<0.005, \ddagger P<0.001$. For details of animals and procedures, see p. 583.

Correlations were studied on the assumption that secondary hyperparathyroidism is pathogenic for osteopenia. There was a positive significant correlation of serum iPTH with serum ALP $(r 0.411 ; P<0.005)$ and corrected urinary Crosslaps ${ }^{\mathrm{TM}}$ ( $r$ 0.857; $P<0.001)$ and negative significant correlations with the various BMD parameters $(P<0 \cdot 005)$.

\section{Recovery experiment (15-30 weeks)}

The normal physical growth pattern, biochemical, serum, urinary and faecal parameters and bone densitometric parameters at 15-30 weeks period in group I have been discussed earlier. The study could not be extended beyond 15 weeks due to the high mortality of the rabbits.
At 15 weeks, as compared with group I rabbits, the group III rabbits (recovery group) had significantly lower body weight $(P<0.005$; Fig. 2(A)), serum iP $(P<0.001$; Fig. $2(\mathrm{C}))$, urinary and faecal $\mathrm{Ca}$ excretion $(P<0.005$; Fig. 3(A) and Fig. 3(C) respectively). These rabbits had significantly higher serum iPTH $(P<0.001$; Fig. 2(D)), ALP $(P<0.05$; Fig. 2(E)), calcitriol $\left(P<0.005\right.$; Fig. 2(F)) and urinary Crosslaps ${ }^{\mathrm{TM}}(P<0 \cdot 05$; Fig. 3(B)). There was no evidence of vitamin D deficiency as these rabbits had comparable calcidiol levels (group I 2.4 (SEM 0.4) $v$. group III $2 \cdot 8(\operatorname{SEM} 0 \cdot 2) \mathrm{ng} / \mathrm{ml}$ ).

After supplementing with the Ca-sufficient diet, there was a rapid catch-up in physical growth until 30 weeks of age. However, at 30 weeks, the weight was still significantly lower as compared with rabbits in group I $(P<0.05$; Fig. $2(\mathrm{~A}))$. There was increase in the mobility and improvement in fur quality of 

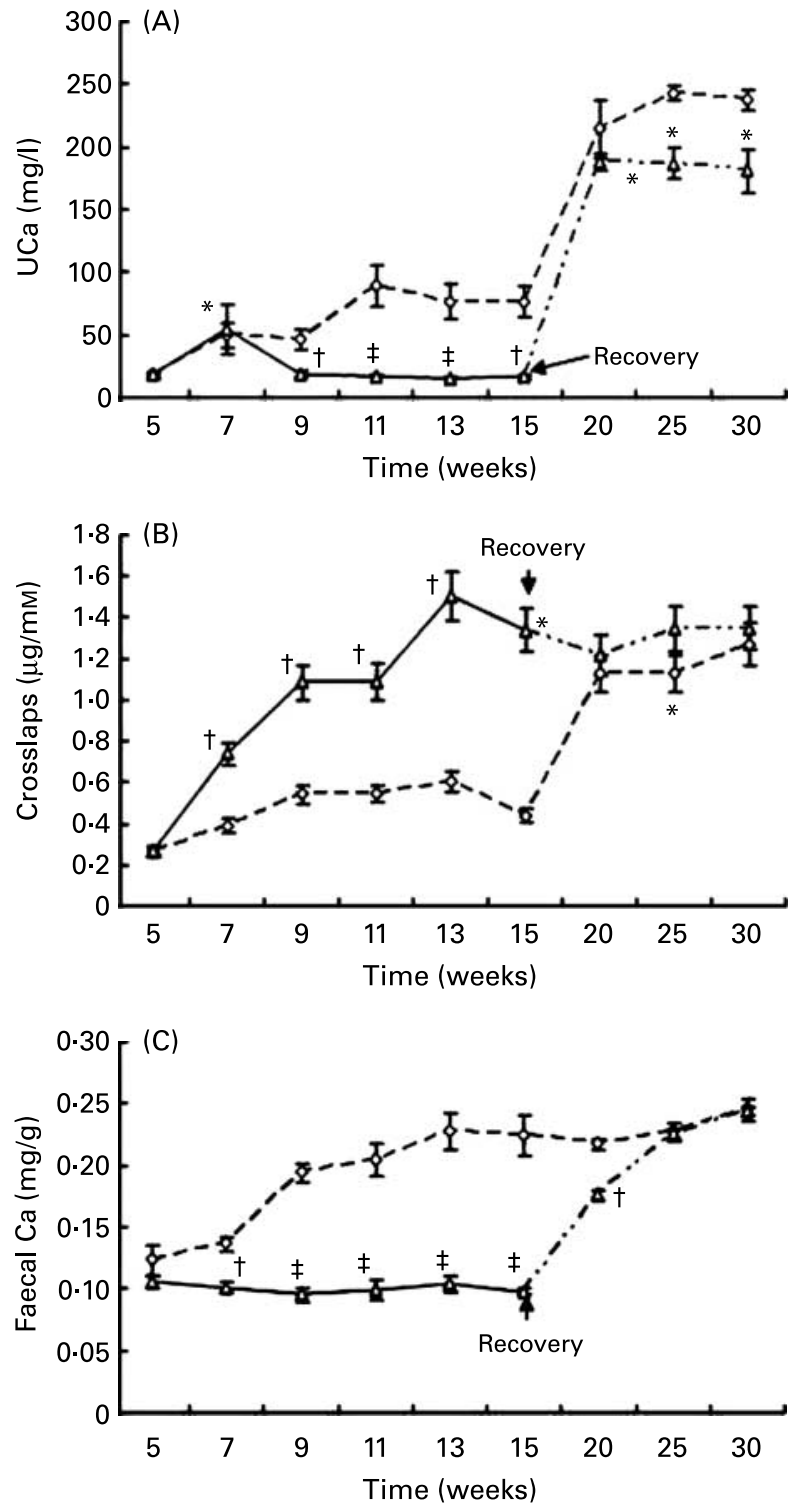

Fig. 3. Serial changes in (A) urinary $\mathrm{Ca}$ excretion (UCa), (B) corrected urinary Crosslaps ${ }^{\mathrm{TM}}$ excretion and (C) faecal Ca excretion in group $1\left(-\diamond_{-}\right)$, group II $(-\downarrow)$ and group III $\left(---_{-}\right)$at different time intervals. Data are expressed as mean values, with their standard errors represented by vertical bars. Mean values were significantly different: ${ }^{*} P<0.05, \quad \dagger P<0.005$, $\ddagger P<0.001$. For details of animals and procedures, see p. 583.

the rabbits' coats. We observed no fractures and mortality in group III rabbits.

There was a progressive increase in serum $\mathrm{CCa}$ and iP levels and faecal $\mathrm{Ca}$ excretion. At 30 weeks, the serum $\mathrm{CCa}$, iP and faecal Ca were comparable ( $P>0.05$; Fig. 2(B), Fig. 2(C) and Fig. 3(C) respectively). We observed a progressive decline in serum iPTH, calcitriol, ALP and urinary Crosslaps ${ }^{\mathrm{TM}}$. These biochemical parameters (i.e. iPTH, calcitriol, ALP, Crosslaps ${ }^{\mathrm{TM}}$ ) were comparable with group I rabbits at 20 weeks, indicating rapid biochemical recovery from secondary hyperparathyroidism. UCa excretion increased steeply at 20 weeks and thereafter remained constant. However, it was significantly lower at 20, 25 and 30 weeks as compared with group I rabbits $(P<0 \cdot 05$; Fig. 3(A)), implying use of dietary $\mathrm{Ca}$ in the mineralisation of bones. There was no significant change in serum calcidiol levels throughout the recovery experiment (30 weeks group I 3.21 (SEM 0.32) v. group III 2.76 (SEM 0.18) $\mathrm{ng} / \mathrm{ml} ; P>0.05$ ). Bone Ca contents in isolated tibia and femora showed a progressive increase until 30 weeks but were still lower than those in group I $(P<0 \cdot 05)$ (data not shown).

The skiagrams of rabbits in group III showed comparable cortical thickness to those of rabbits in group I with no evidence of further fractures.

There was a progressive increase in WBBMD (Fig. 5(A)), WBBMC (data not shown), BMC:weight ratio (data not shown), LSBMD (Fig. 5(B)) and LSBMC (data not shown). Isolated bone in vitro measurements showed a progressive increase in BMD and BMC of whole femur and whole tibia (data not shown). Sub-regional analysis also showed a rapid increase in BMD and BMC in various sub-regions of femur and tibia (Figs. 6 and 7). At 30 weeks, there was no statistical difference in all the densitometric parameters described earlier. The major part of gain in the densitometric parameters was within 5 weeks of the start of the Ca-sufficient diet and thereafter, the gain was less steep.

There was no significant difference observed in body composition analysis (percentage lean mass, percentage fat and percentage BMC) at 20, 25 and 30 weeks in group III rabbits as compared with group I rabbits. No significant correlations were observed.

\section{Discussion}

The present study demonstrates that short-term (10 weeks) administration of a severely Ca-deficient diet $(0.026 \%)$ in weanling male rabbit pups results in hypocalcaemia, hypophosphataemia, elevated serum ALP and calcitriol, hypocalciuria, secondary hyperparathyroidism and increased bone turnover. The gain in BMD at various trabecular and cortical sites is significantly less as compared with those fed with a normal diet. Administration of a normal diet to group II at 15 weeks normalises the metabolic and bone densitometric parameters by 30 weeks of age.

The normal physiological changes in $\mathrm{Ca}$ homeostasis parameters including mean serum Ca, serum iP, total ALP and iPTH in developing male Belgian rabbit pups following weaning until adulthood are in agreement with published reports (Kennedy, 1965; Chapin \& Smith, 1967a,b; Brazy et al. 1980; Gilbert et al. 1980; Buss \& Bourdeau, 1984; Bourdeau et al. 1986; Warren et al. 1989; Gilsanz et al. 1991; Norris et al. 2001). Serum iPTH (human equivalent), calcidiol and calcitriol remained unaltered during development. Lower values of iPTH were observed, possibly due to differences in dietary $\mathrm{Ca}$ intake, species variation, type of PTH analyte measured and differences in antibody in the assay system (Gilsanz et al. 1991; Norris et al. 2004). UCa and Crosslaps ${ }^{\mathrm{TM}}$ excretion data imply that rabbits are hypercalciuric at the age of late development and adulthood. Similar hypercalciuric patterns and high bone turnover have also been reported (Norris et al. 2004).

Rabbits in group I had normal growth despite markedly lower serum calcidiol and calcitriol levels as compared with previous studies (Buss \& Bourdeau, 1984; Warren et al. 1989). In both of the studies, the rabbit diet contained vitamin $\mathrm{D}$ as animal sterol in feed. However, normal growth and reproduction have also been observed with vitamin D content of only $0.28 \mathrm{IU} / \mathrm{g}$ feed (Rockland Rabbit Ration; Harlan Teklad Inc., Madison, WI, USA; 


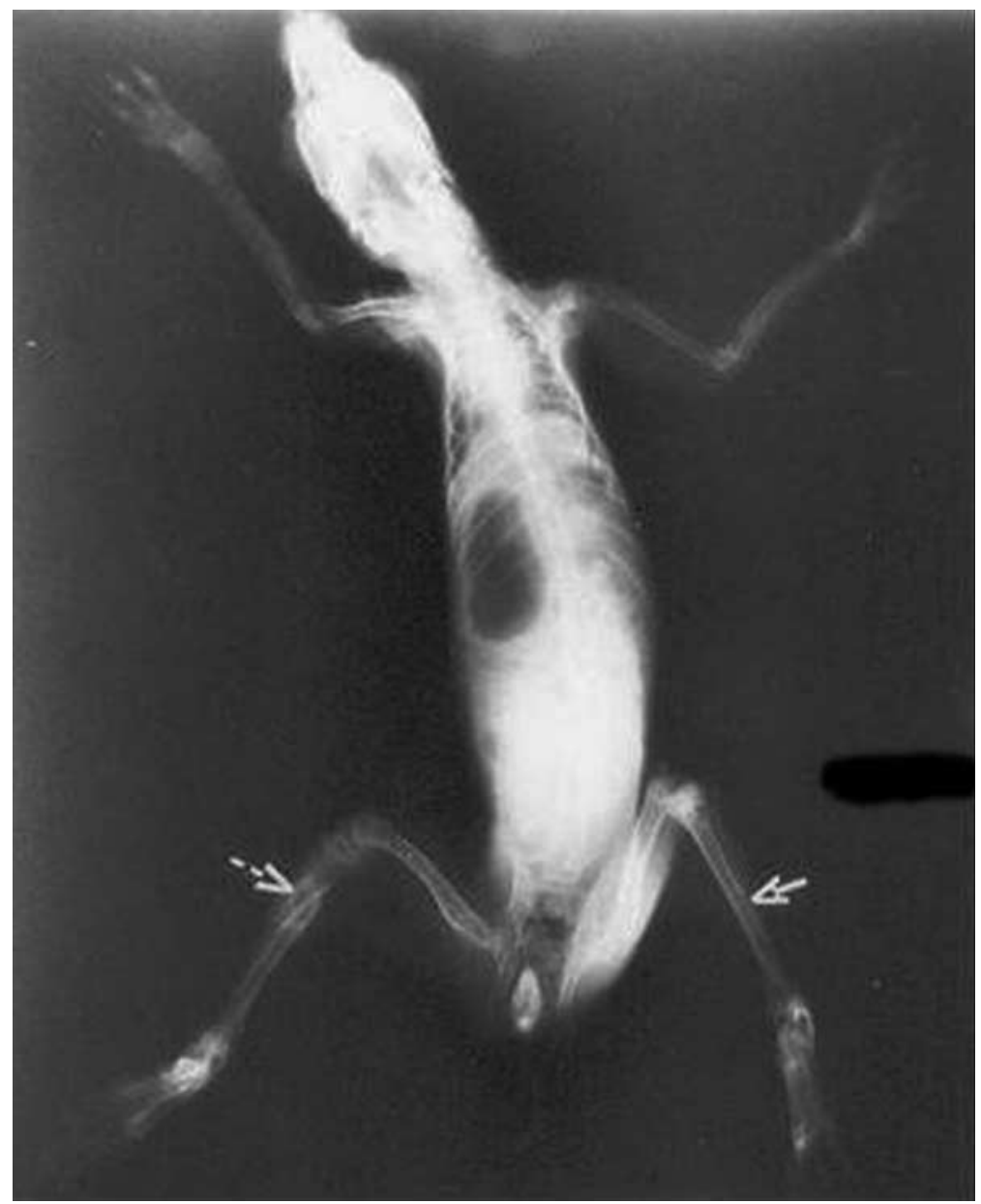

Fig. 4. Skiagram of a Ca-deficient rabbit at 15 weeks showing thinning of the cortical lining $(\longleftarrow)$ and the complete fracture present in the long bone $(--->)$.

analysis, assay, and growth bulletin for Rockland Rabbit Ration, 1970).

The present study is the first to use DXA in measurement of longitudinal changes in BMD in male rabbit pups in a wide range of the post-weaning period. This along with a similar database on female rabbits in the age group of 20-56 weeks reported recently fulfils the need of normative data (Norris et al. 2004). Small animal software was used for measuring BMD, which has shown reasonable short-term percentage $\mathrm{CV}$ in measurements in vivo and in isolated femora and tibia in vitro. A progressive increase in WBBMD, WBBMC, WBBMC:weight ratio, aBMD and $\mathrm{BMC}$ at the LS, femora (total, neck, proximal, mid and distal third), aBMD and BMC at tibia (total, proximal, mid and distal third) was observed until adulthood. Previous studies using quantitative computerised tomography for BMD at the LS $\left(\mathrm{L}_{1}-\mathrm{L}_{5}\right)$ in growing rabbits show a similar pattern as the present study (Gilsanz et al. 1988, 1991). Peak BMD was observed at the global region, proximal and distal femur and femoral neck at 25 weeks and at mid-femur and mid-tibia, primarily cortical sites, at 30 weeks. It was difficult to comment on distal tibia and proximal tibia as increases in BMD more than $2 \%$ continued at 30 weeks. Further follow-up study beyond 30 weeks is required to determine peak BMD at various sites in adult rabbits. Peak BMD at the LS is achieved at 32 weeks (Gilsanz et al. 1991) and 36 weeks (Norris et al. 2004). Compared with the recent report, the lower BMD at the LS (L1-L7) at 20 weeks in our animals is possibly due to species difference although it correlates well with low body weight.

The low-Ca diet induced significant hypocalcaemia, hypophosphataemia, elevated ALP, and iPTH, suggestive of secondary hyperparathyroidism despite no decline in serum calcidiol. Severe Ca deficiency in group II was supported by a significant decline in urinary and faecal $\mathrm{Ca}$ excretion. A similar biochemical picture has been reported in growing as well as adult animal models (Gilsanz et al. 1991; Peterson et al. 1995; Norris et al. 2001; Iwamoto et al. 2004; Bas et al. 2005). The rise in serum calcitriol was due to elevated iPTH, hypocalcaemia and hypophosphataemia-induced stimulation of renal $1 \alpha$-hydroxylase enzyme (Garabedian et al. 1972; Norris et al. 2001). Our biochemical findings are similar to dietary Ca-deficiency-induced rickets in children from the world over (Pettifor et al. 1978, 1981a,b; Marie et al. 1982; Eyberg et al. 1986; Okonofua et al. 1991; Bhimma et al. 1995; Oginni et al. 1996; Fischer et al. 1999; Balasubramanian et al. 2003; DeLucia et al. 2003; Rajeshwari et al. 2003). Histology and histomorphometric studies have shown osteomalacia (Marie et al. 1982).

We observed a small but significant difference in serum $\mathrm{P}$ between group I and group II at 13 and 15 weeks. Earlier studies 

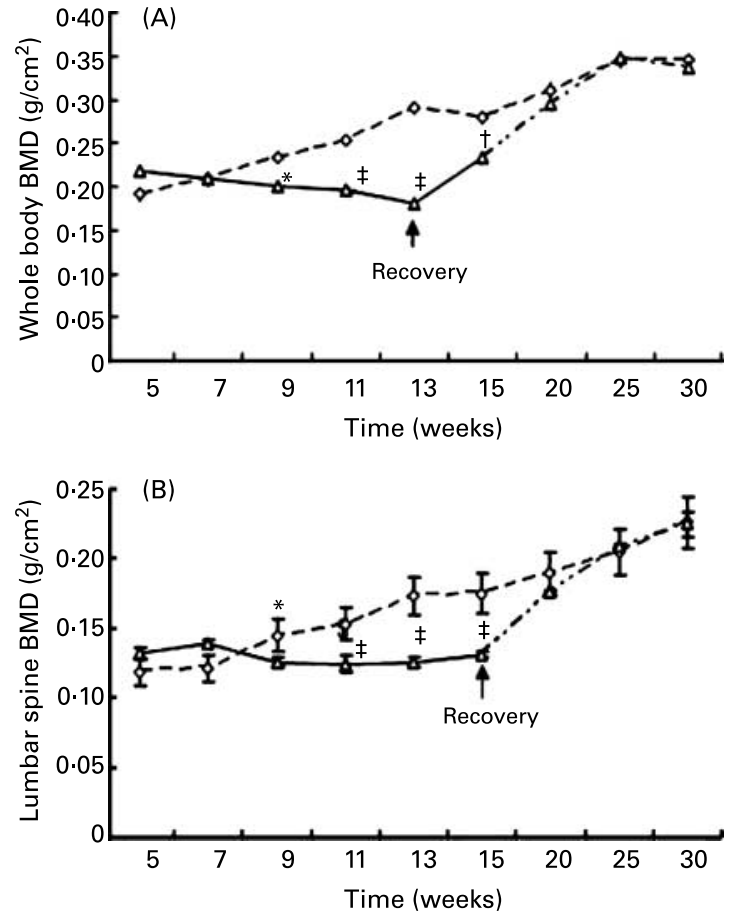

Fig. 5. Serial changes in in vivo bone mineral density (BMD) at (A) whole body and (B) lumbar spine in group $1\left(-\diamond_{-}\right)$, group II $\left(--_{-}\right)$and group III (-- - -) at different time intervals. Data are expressed as mean values, with their standard errors represented by vertical bars. Mean values were significantly different: ${ }^{*} P<0.05, \dagger P<0.005, \ddagger P<0.001$. For details of animals and procedures, see p. 583.
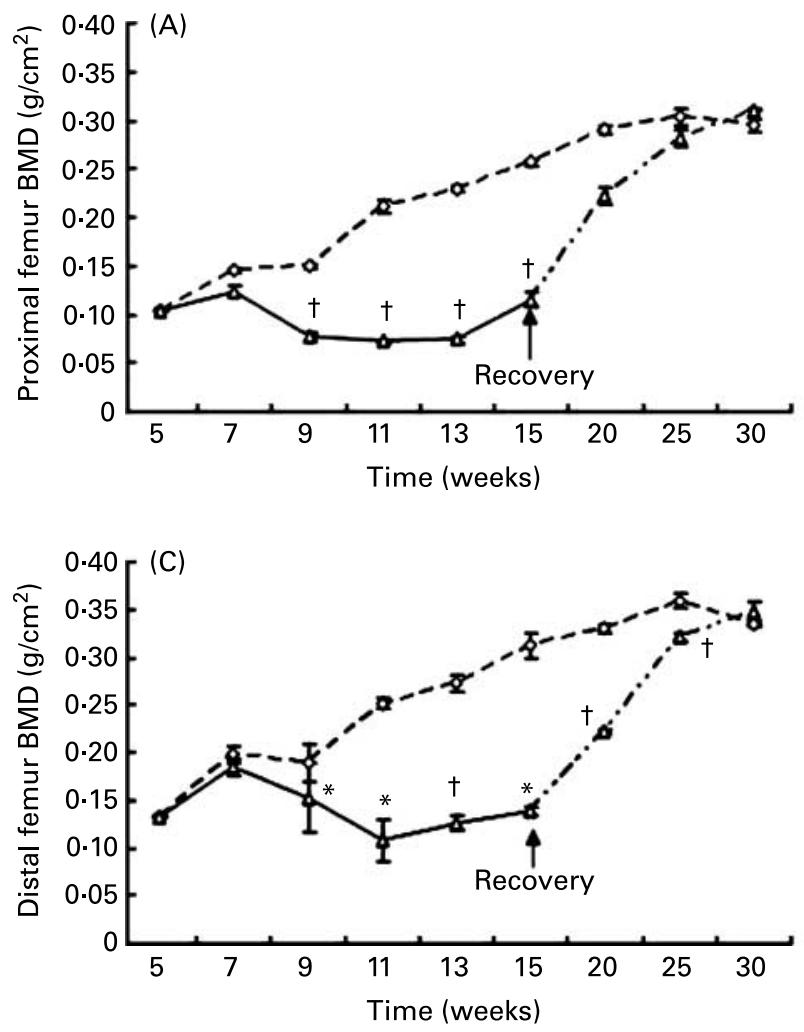

with moderate $\mathrm{Ca}$ deficiency $(0 \cdot 1$ to $0.15 \% \mathrm{Ca})$ have shown no significant difference in serum P (Gilsanz et al. 1991; Iwamoto et al. 2004). Hypophosphataemia occurs in only $55 \%$ of children with Ca-deficiency rickets (DeLucia et al. 2003).

$\mathrm{Ca}$ deficiency results in diffused parathyroid hyperplasia, increased parathyroid weights and percentage of cells in the $\mathrm{S}$-phase and decreased levels of $\mathrm{Ca}$-sensing receptor mRNA in parathyroid tissue (Bas et al. 2005). Examination of parathyroid in group II rabbits would have yielded similar results.

We observed significantly elevated urinary Crosslaps ${ }^{\mathrm{TM}}$ excretion and elevated total serum ALP levels in group II, suggesting increased bone turnover. Children with Ca-deficiency rickets also have elevated collagen turnover, as evidenced by an increase in circulating $\mathrm{N}$ - and $\mathrm{C}$-terminal propeptides (intact PINP and PICP) and C-terminal telopeptide (ICTP) of type I collagen, and N-terminal propeptide (PIIINP) of type III collagen (Sharp et al. 1997; Oginni et al. 2003). Although total serum ALP was elevated in all the cases, serum osteocalcin has been reported to be lower (Sharp et al. 1997) or show no change (Okonofua et al. 1991). However, elevated osteocalcin levels have been observed in a few patients with extremely high serum calcitriol and PTH levels (Okonofua et al. 1991). Serum osteocalcin could not be measured in our experiment due to the non-availability of the relevant antibody.

In rabbits on the Ca-deficient diet, there was significantly less gain in BMD and BMC in the whole rabbit, the LS in vivo, femora and tibia in vitro, both in whole bone and all the sub-regions. The significantly low BMD was observed at midfemur and femur neck regions as early as the 7th week and in all the regions at the 9th week and onwards. Spontaneous
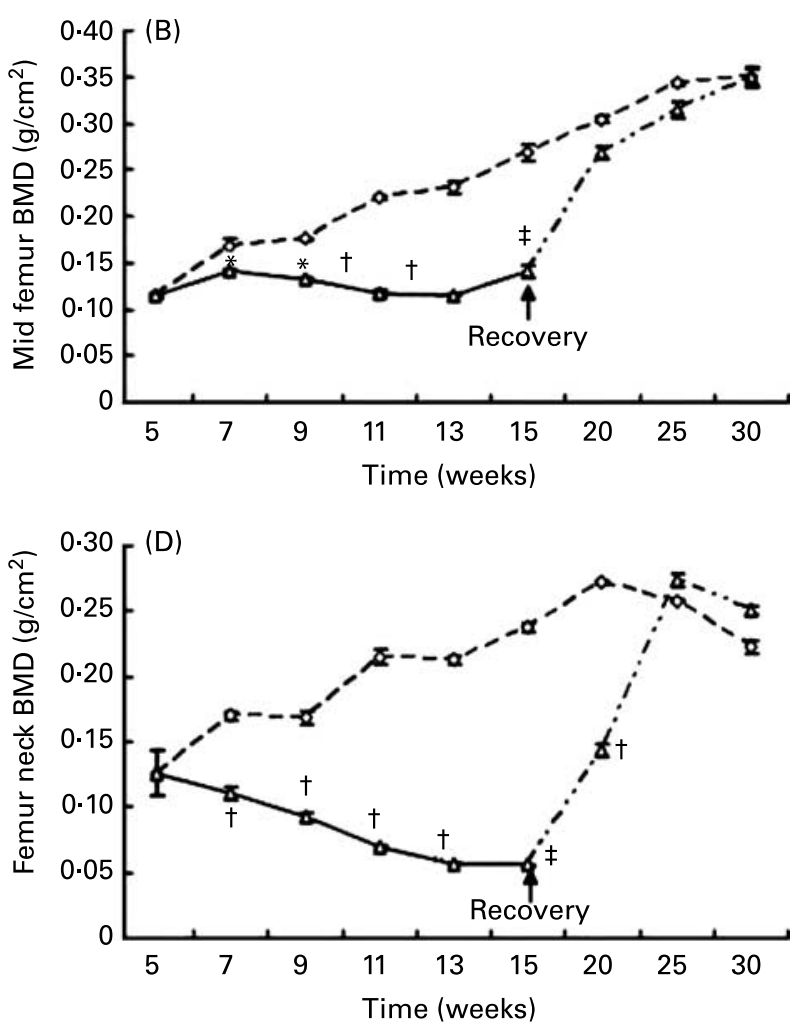

Fig. 6. Serial changes in in vitro bone mineral density (BMD) at (A) proximal femur, (B) mid-femur, (C) distal femur and (D) femur neck in group 1 ( $\diamond-)$, group II $(-\downarrow)$ and group III (-- --$)$ at different time intervals. Data are expressed as mean values, with their standard errors represented by vertical bars. Mean values were significantly different: ${ }^{*} P<0.05, \dagger P<0.005, \ddagger P<0.001$. For details of animals and procedures, see p. 583. 

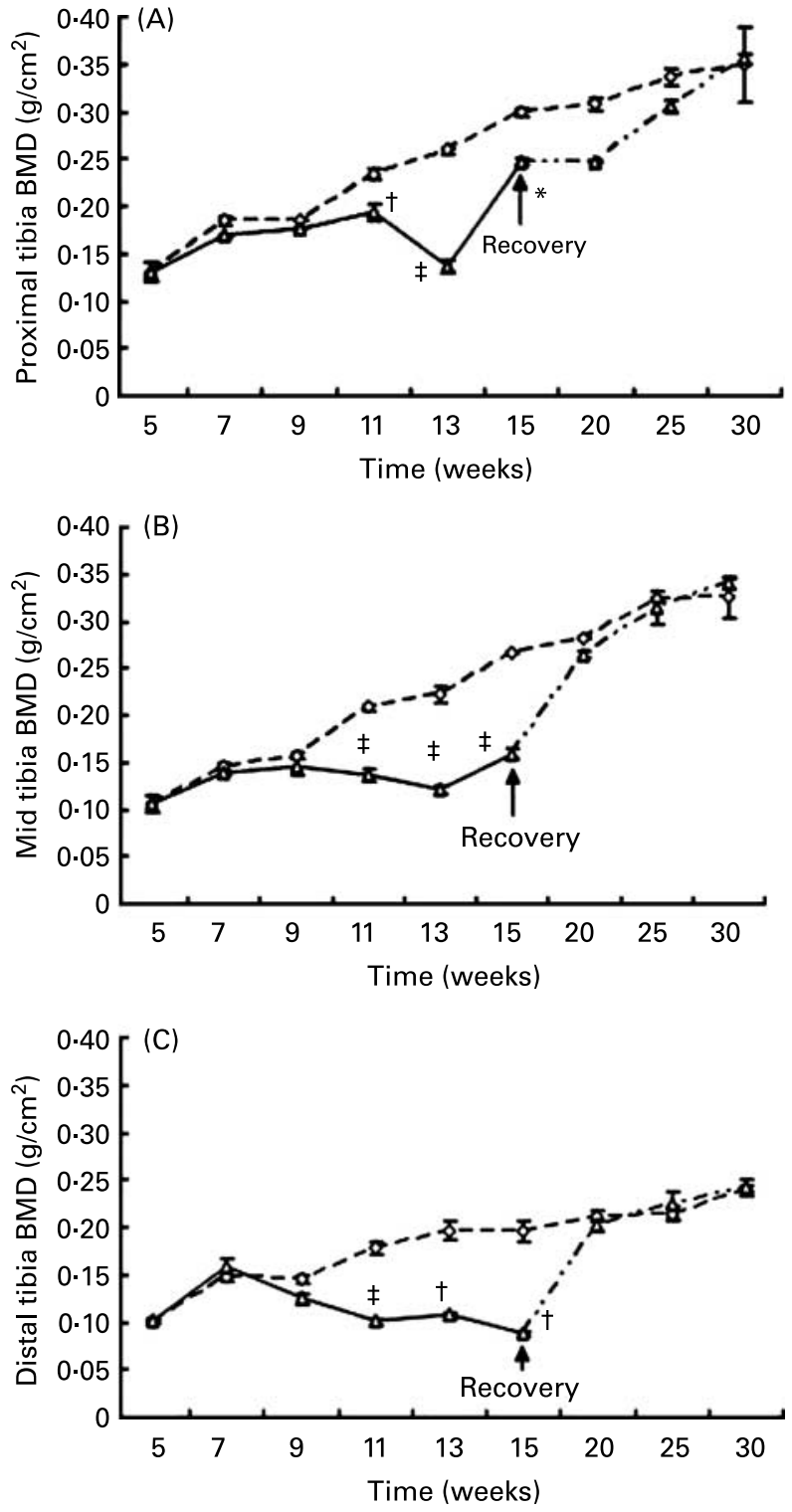

Fig. 7. Serial changes in in vitro bone mineral density (BMD) at $(A)$ proximal tibia, (B) mid-tibia and (C) distal tibia in group $1\left(-\diamond_{-}\right)$, group $\|\left(-\Delta_{-}\right)$ and group III (- $-\downarrow-)$ at different time intervals. Data are expressed as mean values, with their standard errors represented by vertical bars. Mean values were significantly different: ${ }^{\star} P<0.05, \dagger P<0.005, \ddagger P<0.001$. For details of animals and procedures, see p. 583.

fractures in two rabbits suggested reduced tensile strength of long bones. Ca deficiency involved both cortical and trabecular bone in our experimental model. Furthermore, the epiphyseal fusion appeared at about 30-32 weeks in both normal as well as $\mathrm{Ca}$-deficient rabbits. The only published study to date shows poor accrual of BMD at the LS as measured with quantitative computerised tomography in growing rabbits on a Cadeficient diet and histomorphometric evidence for osteopenia and not osteomalacia (Gilsanz et al. 1991). Compromised bone mineralisation and osteopenia have also been noted in the Ca-deficient state in a normocalcaemic animal model, the rat (Thomas et al. 1988). Histomorphometry of lumbar vertebra, femur or tibia might have elaborated the distinction between osteopenia and osteomalacia in the present study.
In recovery experiments, provision of normal dietary $\mathrm{Ca}$ for 15 weeks to Ca-deficient developing rabbit pups reverses the impaired growth, mobility, muscle weakness, hypocalcaemia, secondary hyperparathyroidism, and elevated serum ALP and urinary Crosslaps ${ }^{\mathrm{TM}}$ excretion comparable with control adult rabbits. The faecal $\mathrm{Ca}$ and $\mathrm{UCa}$ excretion rises and there is a fall in serum calcitriol levels, with no changes observed in serum calcidiol levels. The results are similar to the studies of Ca supplementation alone in Ca-deficient rachitic children (Pettifor et al. 1979; Okonofua et al. 1991; Fischer et al. 1999; Oginni et al. 1996, 2003).

There is catch-up gain in BMD in the whole animal in vivo and tibia and femora (whole and sub-regional) in vitro. At 30 weeks, BMD parameters at these sites were comparable with those of rabbits in group I. In vitro Ca content analysis of femur and tibia revealed significant recovery. Thus, normal $\mathrm{Ca}$ intake rapidly reverses the biochemical and BMD parameters in severe $\mathrm{Ca}$ deficiency in developing rabbit pups. However, the bone mineral and $\mathrm{Ca}$ content in the bones, even after recovery, remains significantly lower than that present if the rabbits were not Ca deficient in agreement with Moore et al. (1963). The present study supports the existence of osteopenia and secondary hyperparathyroidism resultant from Ca deficiency. It also emphasises the changes due to $\mathrm{Ca}$ deficiency are reversible. Histomorphometric studies are required to make a definitive diagnosis of osteomalacia. Thus $\mathrm{Ca}$ deficiency-induced osteopenia and secondary hyperparathyroidism should be considered as a distinct clinical entity.

There is only one elegant study available in the literature that describes the effect of $\mathrm{Ca}$ supplementation on growth and development to a Ca-deficient female rat model (Peterson et al. 1995). The present results of dietary Ca levels affecting the peak bone mass are in broad agreement with the result of Peterson et al. (1995). The present results of improved BMD and bone mineral and $\mathrm{Ca}$ content showing positive effect of dietary Ca rehabilitation, though, are in agreement with studies mentioned earlier, in contrast with the negative effect of treatment if instituted during the late development period (Peterson et al. 1995). One may explain the discrepancies based on differences in animal model (rat $v$. rabbit), early institution of rehabilitation, severity of $\mathrm{Ca}$ deficiency, and differences in sex. We would like to reserve comment due to our inability to perform detailed histomorphometry analysis and the absence of a calcitropic hormone profile in Peterson's study, except to say that results from the present study are more in tune with human studies (Oginni et al. 2003). In that respect, the rabbit model apparently serves well as a viable model for human bone physiology (Norris et al. 2001).

In conclusion, short-term severe $\mathrm{Ca}$ deficiency in developing rabbits induces hypocalcaemia, secondary hyperparathyroidism, increased bone turnover in favour of bone resorption and significantly lesser accrual of BMD in the whole animal and in long bones. These changes are reversible with $\mathrm{Ca}$ supplementation if instituted earlier. Further, the rabbit is an ideal hypercalcaemic animal model of Ca-deficiency-induced secondary hyperparathyroidism and BMD changes. But, more studies are required for histomorphometric analysis, tensile strength and fracture potential in adulthood.

\section{Acknowledgements}

An intramural grant (project PGI/DIR/RC/175/1999) from the Sanjay Gandhi Postgraduate Institute of Medical Sciences, Lucknow 226 014, India is gratefully acknowledged. We are 
grateful to Professor Carol Pilbeam, Department of Medicine, University of Connecticut Health Center, Farmington, CT, USA for correcting the manuscript and helpful suggestions. We thank Mr Vivek Saigal for computer artwork.

\section{References}

Balasubramanian K, Rajeshwari J, Govil YC, Agarwal AK, Kumar A \& Bhatia V (2003) Varying role of vitamin D in etiology of rickets in young children vs adolescents in northern India. J Trop Pediatr 49, 201-206.

Bas S, Bas A, Lopez I, Estepa JC, Rodriguez M \& Aguilera-Tejero E (2005) Nutritional secondary hyperparathyroidism in rabbits. Domest Anim Endocrinol 28, 380-390.

Bhimma R, Pettifor JM, Coovadia HM, Moodley G \& Adhikari M (1995) Rickets in black children beyond infancy in Natal. S Afr Med J 85, 668-672.

Bourdeau JE, Schwer-Dymerski DA, Stern PH \& Langman CB (1986) Calcium and phosphorous metabolism in chronically vitamin D deficient laboratory rabbits. Miner Electrolyte Metab 12, 176-185.

Brazy PC, McKeown JW, Harris RH \& Dennis VW (1980) Comparative effects of dietary phosphate, unilateral nephrectomy and parathyroid hormone on phosphate transport by rabbit proximal tubule. Kidney Int 17, 788-800

Buss SL \& Bourdeau JE (1984) Calcium balance in laboratory rabbits. Miner Electrolyte Metab 10, 127-132.

Chapin RE \& Smith SE (1967a) Calcium requirements of growing rabbits. Lab Anim Sci 26, 67-71.

Chapin RE \& Smith SE (1967b) The calcium tolerance of growing and reproducing rabbits. Cornell Vet 57, 480-491.

DeLucia MC, Mitnick ME \& Carpenter TO (2003) Nutritional rickets with normal circulating 25-hydroxyvitamin $\mathrm{D}$ : a call for reexamining the role of dietary calcium intake in North American infants. J Clin Endocrinol Metab 88, 3539-3549.

Eyberg C, Pettifor JM \& Moodley G (1986) Dietary calcium intake in rural black South African Children: the relationship between calcium intake and calcium nutritional status. Hum Nutr Clin Nutr 40C 69-74.

Fischer PR, Rahman A, Cimma JP, et al. (1999) Nutritional rickets without vitamin D deficiency in Bangladesh. J Trop Paediatr 45, 291-293.

Garabedian M, Holick MF, DeLuca HF \& Boyle IT (1972) Control of 25hydroxycholecalciferol metabolism by parathyroid glands. Proc Natl Acad Sci USA 69, 1673-1676.

Gilbert PJ, Schlondorff D, Trizna W \& Fine LG (1980) Renal effects of parathyroid hormone in the rabbit. Miner Electrolyte Metab 3, 291-301.

Gilsanz V, Roe TF, Antunes J, Carlson M, Duarte ME \& Goodman WG (1991) Effect of dietary calcium on bone density in growing rabbits. Am J Physiol 260, E471-E476.

Gilsanz V, Roe TF, Gibbens DT, Schulz EE, Carlson ME, Gonzalez O \& Boechat MI (1988) Effect of sex steroids on peak bone density of growing rabbits. Am J Physiol 255, E416-E421.

Iwamoto J, Takeda T, Sato Y \& Yeh JK (2004) Response of cortical and cancellous bones to mild calcium deficiency in young growing female rats: a bone histomorphometry study. Exp Anim 53, 347-354.
Kennedy A (1965) The urinary excretion of calcium by normal rabbits. J Comp Pathol 75, 69-74.

Marie PJ, Pettifor JM, Ross FP \& Glorieux FH (1982) Histological osteomalacia due to dietary calcium deficiency in children. $N$ Engl J Med 307, 584-588.

Moore T, Impey SG, Martin PEN \& Symonds KR (1963) Meat diets. II. Effect of the age of rats on their ability to withstand the low calcium intake induced by a diet of minced beef. J Nutr 80, 162-170.

Norris SA, Pettifor JM, Gray DA \& Buffenstein R (2001) Calcium metabolism and bone mass in female rabbits during skeletal maturation: effects of dietary calcium intake. Bone 29, 62-69.

Oginni LM, Sharp CA, Badru OS, Risteli J, Davie MWJ \& Worsfold M (2003) Radiological and biochemical resolution of nutritional rickets with calcium. Arch Dis Child 88, 812-817.

Oginni LM, Worsfold M, Oyelami OA, Sharp CA, Powell DE \& Cavie MWJ (1996) Etiology of rickets in Nigerian children. J Paediatr 128, 692-694.

Okonofua F, Gill DS, Alabi ZO, Thomas M, Bell JL \& Dandona P (1991) Rickets in Nigerian children: a consequence of calcium malnutrition. Metabolism 40, 209-213.

Peterson CA, Eurell JA \& Erdman JW Jr (1995) Alterations in calcium intake on peak bone mass in the female rat. $J$ Bone Miner Res 10, 81-95.

Pettifor JM \& Moodley G (1997) Appendicular bone mass in children with high prevalence of low dietary calcium intakes. J Bone Miner Res 12, 1824-1832.

Pettifor JM, Ross FP, Travers R, Glorieux FH \& DeLuca HF (1981a) Dietary calcium deficiency: a syndrome associated with bone deformities and elevated serum 1,25-dihydroxyvitamin D concentrations. Metab Bone Rel Res 2, 301-305.

Pettifor JM, Ross P, Moodley G \& Shuenyane E (1979) Calcium deficiency in rural black children in South Africa - a comparison between rural and urban communities. Am J Clin Nutr 32, 2477-2483.

Pettifor JM, Ross P, Moodley G \& Shuenyane E (1981b) The effect of dietary calcium supplementation on serum calcium, phosphorus, and alkaline phosphatase concentrations in a rural black population. Am J Clin Nutr 34, 2187-2191.

Pettifor JM, Ross P, Wang J, Moodley G \& Couper-Smith J (1978) Rickets in children of rural origin in South Africa: is low dietary calcium a factor? J Pediatr 92, 320-324.

Rajeshwari J, Balasubramanian K, Bhatia V, Sharma VP \& Agarwal AK (2003) Aetiology and clinical profile of osteomalacia in adolescent girls in northern India. Natl Med J India 16, 139-142.

Sharp CA, Oginni LM, Worsfold M, Oyelami OA, Risteli L, Risteli J \& Davie MWJ (1997) Elevated collagen turnover in Nigerian children with calcium deficiency rickets. Calcif Tissue Int 61, 87-94.

Thomas ML, Ibarra MJ, Solcher B, Wetzel S \& Simmons DJ (1988) The effect of low dietary calcium and calcium supplementation on calcium metabolism and bone in the immature, growing rat. Bone Miner 4 , $73-82$.

Warren HB, Lausen NCC, Segre GV, El-Hajj G \& Brown EM (1989) Regulation of calcitrophic hormones in vivo in the New Zealand white rabbit. Endocrinology 10, 2683-2690. 